\title{
Experiencia en los Derechos Humanos y Bienestar Social, Preparatoria Agrícola y Propedéutico de la Universidad Autónoma Chapingo, México
}

\author{
Experience in Human Rights and Social Welfare in the \\ Agricultural High School and Propaeudetic Program of \\ Universidad Autónoma Chapingo, México
}

\author{
Martha Castillo Beltrán \\ México \\ casbel4@hotmail.com
}

Recibido: 16/05/2018 Aceptado: 31/08/2018

Resumen. El mundo se enfrenta a una extensa diversidad de innovaciones sociales: globalización, el boom de la nueva economía basada en el conocimiento y el surgimiento de una ciudadanía activa y reflexiva, la rápida transmisión de la información disuelve tradiciones y costumbres, imponiendo un enfoque más activo y abierto de la vida. El sistema educativo nacional enfrenta el aumento de las desigualdades sociales, particularmente en materia de acceso al bienestar social y a los derechos humanos (la complejidad social, política, económica y cultural de su establecimiento, promoción y defensa). Uno de los retos de la sociedad mexicana es proporcionar educación de calidad a todos los niños que no han logrado acceder a ella, además de la integración y funcionamiento del sistema educativo y la formación de los ciudadanos responsables, solidarios, participativos y críticos que una democracia moderna requiere (Padilla, 2013). En este estudio se analizó la calidad de la formación de los estudiantes en derechos humanos $(\mathrm{DH})$ y bienestar social (educación, medio ambiente, valores, tolerancia, no discriminación, migración, pobreza, violencia de género, violencia psicológica, etc.), en la Preparatoria Agrícola (PA) y del Propedéutico de la Universidad Autónoma Chapingo. Los estudiantes de esta universidad en su mayoría provienen de zonas altamente marginadas y actåualmente no se han creado materias de esta índole. Se encontró que los alumnos de la PA y del Propedéutico tienen en su mayoría algún concepto de $\mathrm{DH}$, de valores en casa, de respeto, armonía, solidaridad, creen que en la 
ReVista UniversidAd en DiÁlogo • Vol. 9, N. ${ }^{\circ}$ 1, Enero-Junio, 2019 • 31-46

ISSN 2215-2849 • EISSN: 2215-4752

URL: http://www.revistas.una.ac.cr/index.php/dialogo/index CORREO ELECTRÓNICO: universidadendialogo@una.cr

DOI: https://doi.org/10.15359/udre.9-1.2

escuela son influenciados por malas amistades y la mayoría han sufrido en alguna etapa de su vida discriminación, principalmente por ser de bajos recursos, por ser indígenas, por su vestimenta. En general, a los profesores, estudiantes y administrativos no les interesan estos temas, no son tolerantes y se habla poco de $\mathrm{DH}$, no hay materias que impartan temas de DH y de bienestar social (valores, del medio ambiente, tolerancia, educación, migración, no discriminación, etc.). La mayoría de los estudiantes tienen familiares que han migrado a otros estados de la república mexicana o a Estados Unidos, por falta de recursos y para tener una mejor calidad de vida. Creen que inculcar y enseñar los DH en las comunidades más pobres o de indígenas los ayudaría mucho a protegerse y no sufrir de discriminación y abusos.

Palabras claves: bienestar social, derechos humanos, educación, estudiantes

Abstract. The world is facing a wide diversity of social innovation: globalization, the "boom" of the new economy based on knowledge, and the emergence of active and thoughtful citizens. The rapid transmission of information dissolves traditions and customs to impose a more active and open life approach. The national education system faces increasing social inequalities, particularly in the area of access to social welfare and human rights (the social, political, economic, and cultural complexity of its establishment, promotion, and defense). One of the challenges of Mexican society is to provide quality education to all children who have not been able to access it, the integration and functioning of the educational system, as well as the formation of responsible, supportive, participatory, and critical citizens required in a modern democracy (Padilla, 2013). This paper examines the following: training and quality of performance of the students from the agricultural high school (PA) and propaeudeutic program of the Universidad Autónoma Chapingo, Mexico, on education in human rights and social welfare (education, environment, values, tolerance, discrimination, migration, poverty, gender-based violence, psychological issues, etc.). These students mostly come from highly marginalized areas, and currently, no such subjects or courses have been created for them. It was found that students from the PA and propaedeutic program have, mostly, some concept of human rights, values at home, respect, harmony, and solidarity, among others. They believe that, at school, they are influenced by bad friendships, and most have suffered discrimination at some stage in their life, mainly because of clothing, bullying, being indigenous, and having low income to live on. In general, teachers, students, and administrative workers are not interested in these issues; they are non-tolerant people and speak little about human rights. There are no subjects or courses about human rights and problems of social welfare (values, tolerance, environment, migration, education, discrimination, etc.). Most of the students have relatives who have migrated to other states of the Mexican Republic or the USA, in search of resources and a better quality of life. These students also believe that inculcating and teaching human rights values in the 
URL: http://www.revistas.una.ac.cr/index.php/dialogo/index

CORREO ELECTRÓNICO: universidadendialogo@una.cr

DOI: https://doi.org/10.15359/udre.9-1.2

poorest communities or indigenous people would help them protect and not suffer from discrimination and abuse.

Keywords: social welfare, human rights, education, students.

\section{Introducción}

En pleno siglo XXI, el mundo se enfrenta a una extensa diversidad de innovaciones sociales, la rápida transmisión de la información rechaza las tradiciones y costumbres imponiendo un enfoque más activo y abierto de la vida. Desafortunadamente, las innovaciones sociales no van por el mismo camino del bienestar social.

El sistema educativo nacional enfrenta hoy diversos retos que, de no atenderse en el mediano plazo, colaborarán en el aumento de las desigualdades sociales, particularmente en materia de acceso al bienestar. Uno de los retos de la sociedad mexicana es proporcionar educación de calidad a todos los niños y niñas que no han logrado acceder a ella, además de la integración y funcionamiento del sistema educativo y la formación de los ciudadanos responsables, solidarios, participativos y críticos que una democracia moderna requiere (Padilla, 2013). En el caso de México, en cuanto a la tarea de educar en derechos humanos (DH), el Gobierno y los integrantes del poder legislativo se han empeñado en incorporar los compromisos internacionales en materia de educación en DH en la legislación vigente, particularmente en la Constitución Política de los Estados Unidos Mexicanos y en la Ley General de Educación (incluyendo algunas leyes aprobadas para prevenir la discriminación y la violencia, entre otras problemáticas).

En este sentido, este artículo analiza la calidad de la formación de los estudiantes de la Preparatoria Agrícola (PA) y del Propedéutico de la Universidad Autónoma Chapingo, sobre educación en DH y bienestar social (educación, medio ambiente, valores, tolerancia, no discriminación, migración, pobreza, violencia de género, violencia psicológica, etc.), temas sobre los que aún no se han creado materias. En 1995, se implementó el Plan de Estudios, se propuso una educación integral: humanista, desarrollo de habilidades y capacidades intelectuales, juicio crítico y creativo, poniendo énfasis en su desarrollo afectivo en cuanto a intereses, actitudes y valores (Mata y Villar, 1993). En lo que respecta al Propedéutico, en 2011, el cambio de Plan de Estudios fue para favorecer al alumno con mayor tiempo para actividades de formación personal y humanística; no se cumplió. 
ReVISTA Universidad EN DiÁlogo • Vol. 9, N. ${ }^{\circ}$ 1, Enero-Junio, 2019 • 31-46

ISSN 2215-2849 • EISSN: 2215-4752

URL: http://www.revistas.una.ac.cr/index.php/dialogo/index CORREO ELECTRÓNICO: universidadendialogo@una.cr DOI: https://doi.org/10.15359/udre.9-1.2

Actualmente, bajo este contexto educativo, el perfil de ingreso a la PA (ciclo de tres años) es cerca de 1138 alumnos, entre las edades de catorce y dieciséis años (61.7\% hombres y $38.3 \%$ mujeres) y en el Propedéutico (ciclo de un año) 916 alumnos, con edades entre los diecisiete y los diecinueve años (65.2\% hombres y $34.8 \%$ mujeres) (Unidad de Planeación, Organización y Método (UPOM), 2010). Aunado a la condición económica y social que vive el estudiante, así como su capital cultural, que proviene de diferentes poblaciones de la república, cada uno con su propia cultura y lenguaje diferente (48 idiomas); un 25.1\% son indígenas (Servicios Escolares, 2016) y entran a un proceso de formación donde se les ofrece alimentación, habitación y servicios asistenciales mínimos que no se presentan en ninguna parte del país.

Además de sufrir la separación temprana de sus padres y hermanos, aún se encuentran en una etapa importante de su formación y desarrollo, se encuentran solos, les faltan límites y autodisciplina, y la alta exigencia académica y la falta de apoyo emocional provocan baja eficiencia terminal: un $50 \%$ en los estudiantes de la PA y un 40\% en los del Propedéutico (Hernández, 2013).

Cabe mencionar que cerca de la tercera parte del ingreso del 2010, tanto en la PA (32.1\%) como en el Propedéutico (32.3\%), viene de Chiapas, Guerrero y Oaxaca, en estas zonas el grado de marginación es muy alto (Pérez, Livera y Carranza, 2010). Esto muestra una considerable desigualdad en los niveles de desarrollo entre los estados, es decir que hace evidente el nivel de carencias con que viven sus habitantes en cuanto a la falta de acceso a la educación, habitan en viviendas inadecuadas, perciben ingresos insuficientes y residen en localidades pequeñas. El $23.4 \%$ del ingreso a la Preparatoria y casi la tercera parte del ingreso al Propedéutico, $32.4 \%$, procedió de entidades con grado de marginación alto. Estas entidades fueron: San Luis Potosí, Hidalgo, Michoacán, Puebla, Veracruz, Campeche, Tabasco y Yucatán. De los datos anteriores, se pudo observar que más de la mitad del ingreso a la Preparatoria, 55\%, y más de dos quintas partes del ingreso al Propedéutico, $64.7 \%$, procedieron de entidades de muy alta y alta marginación (Pérez, Livera y Carranza, 2010), donde la desigualdad de oportunidades de participación en el proceso de desarrollo y el disfrute de sus beneficios puso en clara situación de desventaja social a proporciones significativas de sus habitantes (CONAPO, 2010).

El CONAPO (Consejo Nacional de Población) define la marginación como un fenómeno estructural múltiple que valora dimensiones, formas e intensidades de exclusión del proceso de desarrollo y disfrute de sus beneficios, expresando 
URL: http://www.revistas.una.ac.cr/index.php/dialogo/index

CORREO ELECTRÓNICO: universidadendialogo@una.cr

DOI: https://doi.org/10.15359/udre.9-1.2

la dificultad para trascender el progreso en el conjunto de la estructura productiva, pues excluye a ciertos grupos sociales del goce de beneficio que otorga el proceso de desarrollo (CONAPO, 2010).

En la Universidad de Chapingo los DH y el bienestar social tienen diversas expresiones histórico-culturales, siendo este un problema fundamental, por lo que debe promoverse la corrección de estructuras y tendencias políticas, económicas y sociales que no permiten el establecimiento de condiciones reales de dignidad e igualdad de las personas. En consecuencia, son una perspectiva crítica de la educación, por lo tanto, la multiplicidad de rasgos humanos y elementos valorativos que reúnen los DH requieren de una comprensión amplia. Los derechos humanos son los que las personas tienen por su calidad humana (Hernández y Fuentes, 1991), se necesita además crear la cultura de los $\mathrm{DH}$, convertirlos en creencias, actitudes y valores de los individuos, expresarlos en las estructuras sociales, políticas y económicas.

Para conocer sobre las experiencias de los estudiantes y sus saberes sobre derechos humanos (DH) y bienestar social (educación, medio ambiente, valores, tolerancia, no discriminación, migración, pobreza, violencia de género, violencia psicológica, etc.). El método que se utilizó fue aplicar un cuestionario-entrevista para investigar acerca de los DH y del bienestar social; los alumnos fueron seleccionados al azar, participaron veintiséis alumnos de primer año, veintidós alumnos de segundo año y veintidós alumnos del Propedéutico y de la Preparatoria Agrícola (PA) de la UACh. Además, se realizaron entrevistas grabadas a algunos de los estudiantes de la PA y del Propedéutico, de la especialidad, y estudiantes que hablaran otra lengua.

El objetivo de este estudio es mostrar que los estudiantes de la Preparatoria Agrícola y del Propedéutico utilizan los derechos humanos y viven o disfrutan de un bienestar social. ¿Realmente se aplican en la actualidad estos conceptos, directa o indirectamente, para orientarlos a manifestar y promover la comprensión de la educación, los derechos humanos y sobre todo el bienestar social?

\section{Resultados}

Los alumnos de primer año no tienen un concepto bien definido de los $\mathrm{DH}$, sin embargo, mencionan que son fundamentales para una sociedad, ayudan a desarrollarse, convivir y comprenderse entre ellos, así como para protegerse de algún tipo de discriminación. En cuanto a los valores, opinan que son indispensables, aunque no todos los practican, son el motor que impulsa, el 
Revista UniversidAd en DiÁlogo • Vol. 9, N. ${ }^{\circ}$ 1, Enero-Junio, 2019 • 31-46

ISSN 2215-2849 • EISSN: 2215-4752

URL: http://www.revistas.una.ac.cr/index.php/dialogo/index CORREO ELECTRÓNICO: universidadendialogo@una.cr

DOI: https://doi.org/10.15359/udre.9-1.2

respeto, la tolerancia y la comprensión, al aplicarlos se hace un mundo más justo. Lamentablemente, aluden a que los DH no se aplican correctamente, "se dice que hay justicia, pero no la hay".

Les pedimos que nos hablaran acerca de su estancia en la PA, de sus profesores, compañeros de clase e incluso de los administrativos, si ejercían los DH y los valores. Mencionan que sus compañeros sí cuidan el medio ambiente, tienen tolerancia, educación. Nos señalan que la mayoría de los profesores y compañeros de clase utilizan un lenguaje inapropiado, los profesores no son tolerantes, en especial los profesores de matemáticas, algunos son impuntuales y groseros. Los administrativos no te ponen atención, ni siquiera te saludan, algunos son prepotentes. Por el contrario, algunos observaron que la mayoría de los profesores que ejercen valores, cumplen con normas del entorno y respetan los valores.

Realizaron una reflexión sobre si es en la casa o en la escuela donde se debe hablar y educar sobre valores y actitudes, prevenir la discriminación, promover la igualdad y la participación en la toma de decisiones. El 90\% piensa que es en la casa donde se debe reflexionar sobre estos temas. En casa se aprenden valores, en la escuela refuerzas lo que ya aprendiste en casa, lo pones en práctica. Algunos piensan que en la escuela eres influenciado por malas amistades.

Varios han adoptado algunos conceptos para su vida, como "todos tenemos derechos, el de mi familia", "sin valores el mundo y la sociedad serían peores de lo que ya son", "la igualdad, solidaridad y respeto", "los DH son buenos y saberlos", "los valores se forman desde casa, el respeto", "no discrimines, sé generoso", "lo que respalda al ser humano son sus valores", "son fundamentales en mi vida diaria y me dan crecimiento moral".

$\mathrm{Al}$ averiguar si alguna vez habían sufrido discriminación, 65\% de alumnos de primero señalaron que sí la sufrieron: discriminación verbal y física por ser indígenas y por su color de piel, por ser del estado de Oaxaca, por su vestimenta, por compañeros y hermanos verbal y moralmente, por funcionarios públicos y dueños de negocios; bullying por ser gordo. La reacción de algunos estudiantes ante la discriminación fue llegar a los golpes, la de otros fue sentir tristeza y baja autoestima, o enojo, otros no dijeron nada. Unos reflexionaron sobre que es ilógico que los discriminen por ser indígenas si las raíces de la mayoría de las personas mexicanas provienen de ahí. 
URL: http://www.revistas.una.ac.cr/index.php/dialogo/index

CORREO ELECTRÓNICO: universidadendialogo@una.cr

DOI: https://doi.org/10.15359/udre.9-1.2

Así mismo, opinan que la cultura mexicana posee bienestar social congruente, primero con la tolerancia, raza, discriminación, migración, medio ambiente, en minoría la equidad, el pensamiento, la cultura, los modales y la fidelidad. En contraste, los no congruentes son principalmente la discriminación, migración, raza, medio ambiente, en minoría tolerancia, honestidad y respeto, corrupción política, indiferencia, conflicto.

Se les preguntó si tenían familiares que han migrado a otros estados de la república u otro país. El 58\% de los estudiantes indicaron que sus tíos, primos, hermanos e incluso sus padres han migrado a las ciudades y principalmente a Estados Unidos, han migrado por necesidad económica y para tener una mejor vida.

Ante la pregunta: ¿Crees que trabajar con la gente en las comunidades puede sensibilizarla, ampliar sus conocimientos acerca de los valores, actitudes y habilidades para aplicar y promover los DH en su vida y su trabajo? La mayoría dijo que sí, porque es una forma de conocer y apoyar sus puntos de vista, de ver una perspectiva diferente de la vida, se intercambian cultura y valores. En las comunidades hay poco recurso para estudiar; ver que la realidad es muy dura en las zonas rurales sensibiliza y humaniza, todos necesitamos de todos. Las personas que tienen conocimiento discriminan a las personas de las comunidades, si congeniaran podrían ayudar a la gente rural, ya que algunos son muy cerrados en su forma de pensar.

¿Qué propones para que tus compañeros conozcan los DH? Que los profesores hablen un poco más de los valores y ponerlos en práctica, y que el alumno tenga disposición e interés. Primero deben empezar por respetarse ellos mismos y después aplicarlo a la sociedad. Que salgan a la calle y busquen a todas las personas necesitadas y les den un pedazo de pan. Ser congruentes con los valores, que se vean los diferentes casos en el país en los que se aplican los $\mathrm{DH}$, que se comparen y analicen. Que se haga una campaña para que se conozcan, ponerse en el lugar del otro, cómo se sentirían si a ellos les hicieran lo mismo. También se les preguntó si habían apoyado el pasado 19 de septiembre de este año en el terremoto, diecinueve estudiantes dieron su apoyo e indicaron que lo que los impulsó fue ayudar y pensar en que si les pasara lo mismo les gustaría que los ayudaran; a los otros, por ser menores de edad, no los dejaron participar.

Los alumnos de segundo año tienen un concepto más amplio sobre los $\mathrm{DH}$, señalan que son importantes por sus leyes o derechos, nos protegen y nos identifican como seres racionales e igualitarios, ya que tenemos sociedades 
Revista UniversidAd en DiÁlogo • Vol. 9, N. ${ }^{\circ}$ 1, Enero-Junio, 2019 • 31-46

ISSN 2215-2849 • EISSN: 2215-4752

URL: http://www.revistas.una.ac.cr/index.php/dialogo/index CORREO ELECTRÓNICO: universidadendialogo@una.cr

DOI: https://doi.org/10.15359/udre.9-1.2

regidas por la economía y la jerarquía. Son necesarios para tener un mejor desarrollo ciudadano humanitario y forman personas de bien; una sociedad con $\mathrm{DH}$, valores y respeto tiene una mejor convivencia, más armónica. Son fundamentales, ya que inculcan respeto y aprendes qué puedes y qué no hacer, limitan nuestras acciones, lo moralmente correcto. Sin embargo, no se han implementado métodos para llevarlos a cabo en todo el mundo.

Consideran que los profesores, compañeros y administrativos no dominan estos temas, los estudiantes no los juzgan necesarios, piensan que se deben aprender de niños, no ahora, les importa más pasar las materias. Los profesores y alumnos no tienen respeto mutuo. En cuanto a la educación, los profesores se oponen a nuevas ideas o a que los alumnos expresen las suyas. Muchos profesores y administrativos no son tolerantes y muchas veces son groseros.

En cuanto a la reflexión sobre si es en casa o en la escuela donde se habla y educa sobre estos temas, el $100 \%$ de los estudiantes dijeron que en casa aprendieron a ser respetuosos y a saber decidir, es la principal formación humana, gracias a los valores que les inculcaron sus padres. Han adoptado algunos conceptos como "la tolerancia y el respeto"; "el respeto al derecho ajeno es la paz", "esto lo define todo: todos tenemos los mismos derechos, la igualdad"; "los valores son pensamientos establecidos por las sociedades según sea el caso, para mejorar la convivencia con las personas"; "son reglas o normas que regulan el comportamiento y definen los derechos de cada uno"; "los DH son aquellas libertades y poner límites, solidaridad, tolerancia". También, el 37\% de los estudiantes de segundo año han sufrido discriminación, principalmente por ser de pueblo, provocaciones verbales, bullying por ser gordo; algunos lo tomaron como positivo por la riqueza cultural y mejor ambiente, otros con tristeza y enojo.

Consideran que la cultura mexicana posee bienestar social congruentes, primero con la tolerancia, medio ambiente, discriminación, raza, migración, respeto, solidaridad. Además, piensan que hay poca coherencia y estafeta para liderar a un país, sin la esencia de que son DH. En contraste con los que no son congruentes, principalmente tenemos la discriminación, migración, racial a etnias y morenos, robo, sabotaje, mal trato, la tolerancia es rara en México. En la cultura mexicana hay mucha discriminación hacia los distintos lugares del país, por ejemplo, en la Universidad de Chapingo se dan algunos casos en los que te discriminan los de otros estados por creerse mejor que las personas de tu estado. Todo esto tiene que ver con la poca educación y la falta de moral que nos han llevado al vandalismo y a la delincuencia, la falta de respeto a la ciudadanía y al Gobierno, tanto interno como externo. 
URL: http://www.revistas.una.ac.cr/index.php/dialogo/index

CORREO ELECTRÓNICO: universidadendialogo@una.cr

DOI: https://doi.org/10.15359/udre.9-1.2

Se les consultó a los estudiantes si tienen familiares que han migrado a otros estados de la república u otro país, el $81 \%$ de los estudiantes indicaron que sus tíos, primos, hermanos y padres han migrado principalmente a Estados Unidos, migraron para tener una mejor forma de vivir y por falta de recursos.

$¿$ Crees que trabajar con la gente en las comunidades puede sensibilizarla, ampliar sus conocimientos acerca de los valores, actitudes y habilidades para aplicar y promover los DH en su vida y su trabajo? Sí, porque al estar alejados de la sociedad se privan de sucesos de interés y es importante enseñar todo lo que se sabe, muchos no conocen los DH que tienen y dejan que los discriminen y exploten.

¿Qué propones para que tus compañeros conozcan los DH? Invitar a los compañeros a conocer los derechos humanos y valores y que los respeten, que se informen sobre el tema. Que se deben enseñar desde niños, antes que se forme la personalidad individual. También se les preguntó si habían apoyado en el terremoto, la mayoría ayudó por solidaridad, ayudar no solo a sacar un escombro, sino a su propio pueblo.

Los estudiantes del Propedéutico son alumnos que vienen de otras preparatorias terminadas y cursan un año para entrar a la especialidad. La percepción que tienen sobre DH, valores, etc., es que son fundamentales para la sociedad, sin ellos no habría control de mercado, es la manera ética y moral de comportarnos ante todos, respetando los derechos propios y los de los demás, nos forja la personalidad ante la sociedad para una convivencia tolerante, política, igualitaria, no discriminatoria. Ignorar los $\mathrm{DH}$ ha provocado decadencia en el pueblo de México y lamentablemente la sociedad se aprovecha de ello y no es congruente con lo que dice y hace.

Su estadía en la Universidad ha sido corta, piensan que los temas de DH, valores, medio ambiente y tolerancia son temas de interés, pero a pocos les importa, hoy en día les da igual, no los tocan a profundidad. Creen que los profesores les faltan el respeto a algunos compañeros y a veces a todos, además de ser prepotentes, hay profesores que se apegan a normas y coherencias. La convivencia con los administrativos es buena, aunque de diferentes conveniencias con algunos alumnos.

A su juicio consideran que es en casa donde se llevan a cabo más platicas acerca de los valores y en la escuela se aplican, dan a conocer lo que les enseñaron en casa, reforzándolo; en la escuela solo dan conceptos. Profesan que en casa son mejores los valores que en la escuela y en las comunidades las personas tienen más valores que las de las ciudades. Han salvaguardado algunos valores como 
Revista UniversidAd en DiÁlogo • Vol. 9, N. ${ }^{\circ}$ 1, Enero-Junio, 2019 • 31-46

ISSN 2215-2849 • EISSN: 2215-4752

URL: http://www.revistas.una.ac.cr/index.php/dialogo/index CORREO ELECTRÓNICO: universidadendialogo@una.cr

DOI: https://doi.org/10.15359/udre.9-1.2

"el respeto hacia los demás"; "el derecho al respeto ajeno es la paz"; "todos tus derechos son culminados cuando el derecho del otro comienza"; "todas esas virtudes que tenemos son esenciales en vida". Así mismo, el $48 \%$ ha sufrido discriminación y exclusión, primordialmente por hablar una lengua indígena, por su vestimenta, o bullying por ser reguetonero chaka. Ante la discriminación se han sentido tristes, inseguros; otro dice que agarró a palos a su primo; otro reaccionó a golpes; y otros no dijeron ni hicieron nada, pero se deprimieron.

La apreciación que tienen de la cultura mexicana de la congruencia de bienestar social. La discriminación, raza, tolerancia etc., piensa que tolerancia, raza, migración, medio ambiente, discriminación si son coherentes, se cumple al pie de la letra, pero en general no son correspondientes con las acciones y con lo que se dice. En México no se comportan de manera coherente con los $\mathrm{DH}$, ni se aplican estos derechos, es uno de los países con menos valores, la mayoría no son conscientes de la realidad y cada quien hace lo que quiere. Principalmente somos incongruentes en cuanto a la no discriminación, y el medio ambiente no lo valoramos ni lo respetamos. La tolerancia no es adecuada con los valores, nuestro gobierno no está correctamente establecido. El 100\% de los estudiantes del Propedéutico tienen familias de migrantes, principalmente por la economía del país y la falta de empleo.

Al preguntarles si creen que trabajar con la gente en las comunidades puede sensibilizarla y ampliar sus conocimientos acerca de los valores, actitudes y habilidades para aplicar y promover los DH en su vida y su trabajo, valoran que la cultura en estos lugares es completamente distinta, hay ignorancia, pero se ayudan mutualmente a salir adelante. Al saber sobre DH su mente se abriría, mejoraría la convivencia y se unirían para solucionar problemas. Un estudiante fue tutor comunitario de verano de CONAFE (Consejo Nacional de Fomento Educativo, institución donde se atienden niños y niñas con alto rezago social y marginación, se orienta a poblaciones mestizas, indígenas y migrantes), lugar en el que aprendió a valorar los recursos con los que contaba en su casa y en su comunidad. Otro fue líder de educación comunitaria y vio cómo sus DH eran violados y mal fomentados. Además, en estas comunidades aprendes y conoces personas que están mejor, igual o peor que tú, la experiencia individual te impulsa a encontrar algo mejor. Muchos vivimos en una zona de confort y darse cuenta de que hay personas buenas y malas en la sociedad nos hace despertar sentimientos, nos enseña a ser solidarios, sensibles a la sociedad donde a muchos no les importa nada, solo ellos mismos. 
URL: http://www.revistas.una.ac.cr/index.php/dialogo/index

CORREO ELECTRÓNICO: universidadendialogo@una.cr

DOI: https://doi.org/10.15359/udre.9-1.2

Algunas propuestas para conocer los $\mathrm{DH}$ son primordialmente respetar y no hacer lo que no quieres que te hagan, impartir alguna materia, talleres sociales dentro de la institución, realizar campañas sobre derechos humanos, desde pequeños conocer primero los $\mathrm{DH}$ y reglamentos, con ello construimos nuestro propio ser. En cuanto a si habían apoyado en el terremoto, todos apoyaron, los impulsó la impotencia de ver que perdieron su patrimonio y la satisfacción de ayudar; algunos recaudaron víveres, otros opinan que ayudar es un valor que todos debemos aplicar, la solidaridad. Algunos estudiantes señalan que en sus municipios sufrieron daños, fueron a apoyar y lamentablemente algunos sufrieron robos.

Se llevaron a cabo diez entrevistas de las especialidades (Suelos, Fitotecnia, Mecánica, Economía), donde algunos mencionan que los DH no son temas de interés, sin embargo, durante la carrera les hablan sobre cuidar el medio ambiente y la tolerancia. Creen que los DH son fundamentales para la formación de todos los ciudadanos, algunos tienen la idea de que durante toda su vida han sufrido de bullying por falta de dinero, y de joven es más de juego, según indican algunos, aunque otros no están de acuerdo, ya que hay que poner límites, porque hay insultos y agresiones en el trabajo por pertenecer a otro grupo social. En los sectores más pobres hay muchos malandrines que no estudian, no tienen valores ni educación, suelen ser violentos y te molestan. Los profesores, administrativos y compañeros en la Universidad tratan de utilizar los DH, pero no son tolerantes en la especialidad, si no les caes bien te excluyen, tienes que entrar a su régimen político. El mexicano es solidario, pero le gusta molestar al prójimo, humillarlo, son burlones. Muchas veces no somos congruentes, no nos apoyamos, excepto con el terremoto, en la calle tratas de ayudar, pero cuando se trata de asaltos y necesitas ayuda no te apoyan.

Se entrevistó a tres indígenas que mencionaron que en estos tiempos la ley general de lingüística les llama lenguas indígenas originarias a sus idiomas. Uno de los entrevistados habla zapoteca, y señalaba que sí han sufrido discriminación y burla, se sienten inferiores, la gente se aprovecha y aprecian y tratan mejor a los europeos y norteamericanos y a los indígenas los desvaloran. Los otros dos entrevistados hablan xigue, han tenido problema por el plagio de su vestimenta con los franceses, hace un año, pero las autoridades no hicieron nada, ni le dieron la importancia y no se llevó a cabo la demanda por ser indígenas. La Universidad tiene un espacio donde tratan problemas de discriminación y se apoyan para no tener dificultades. 
Revista UniversidAd en DiÁlogo • Vol. 9, N. ${ }^{\circ}$ 1, Enero-Junio, 2019 • 31-46

ISSN 2215-2849 • EISSN: 2215-4752

URL: http://www.revistas.una.ac.cr/index.php/dialogo/index CORREO ELECTRÓNICO: universidadendialogo@una.cr

DOI: https://doi.org/10.15359/udre.9-1.2

\section{Discusión}

No es fácil definir los DH, cada uno y cada tipo expresa elementos o aspectos del descubrimiento del ser humano, de la profundidad de su riqueza, de aquí la complejidad social, política, económica y cultural de su establecimiento, promoción y defensa.

Por otro lado, Barba (2013) nos señala que los DH no tienen el mismo significado y la misma consecuencia en diversas sociedades. En países desarrollados con una perspectiva progresista de orientación liberal tienden a subordinarlos al modelo capitalista de desarrollo económico, mientras que en los países con menor desarrollo o pobres los DH enfrentan situaciones críticas de legitimidad y vigencia. De todos modos, aun en los países desarrollados existen problemas de legitimidad y respeto de los $\mathrm{DH}$, como se manifiesta en los problemas de los inmigrantes, el racismo, etc.

Los estudiantes de la PA y del Propedéutico tienen en su mayoría algún concepto de $\mathrm{DH}$, los de primer año más de valores en casa, de respeto, armonía, solidaridad. Algunos piensan que en la escuela eres influenciado por malas amistades. Los de segundo y del Propedéutico incluyen términos de economía, política, ética y moral, racionalidad, igualdad, no discriminación, y señalan que existen leyes o derechos que los protegen, pero tenemos sociedades regidas por la economía y jerarquía en el país y el mundo. Opinan que los DH en México no se aplican, y la mayoría ha sufrido en alguna etapa de su vida discriminación, principalmente por ser de bajos recursos, indígenas (etnias), vestimenta, migrante, por ser morenos, robo, sabotaje, maltrato; la tolerancia es rara en México. Todo esto debido a la poca educación y la falta de moral que nos han llevado al vandalismo y a la delincuencia, la falta de respeto a la ciudadanía y al Gobierno, tanto interno como externo.

En general, su reacción ante la discriminación fue de enojo, o una sensación de baja autoestima, sentirse menos y tristes, algunos reaccionaron con agresiones. Habitualmente a los profesores, estudiantes y administrativos no les interesan estos temas, no son tolerantes y hablan poco de $\mathrm{DH}$, no hay materias que den estos temas sobre DH y bienestar social (valores, medio ambiente, tolerancia, educación, migración, no discriminación, etc.). La mayoría de los estudiantes tienen familiares que han migrado a otros estados de la república mexicana o a Estados Unidos por falta de recursos y para tener una mejor calidad de vida. Creen que inculcar y enseñar los DH en las comunidades indígenas, empobrecidas y marginadas les ayudaría mucho a protegerse y no sufrir de discriminación y abusos. Se cree que en la cultura mexicana existen congruencias con el 
URL: http://www.revistas.una.ac.cr/index.php/dialogo/index

CORREO ELECTRÓNICO: universidadendialogo@una.cr

DOI: https://doi.org/10.15359/udre.9-1.2

bienestar social, pero no se cumplen al pie de la letra en cuanto a los $\mathrm{DH}$; no son coherentes con las acciones y con lo que se dice, no se comportan de manera paralela a los DH, ni se aplican estos derechos. México es uno de los países con menos valores, la mayoría no son conscientes de la realidad y cada quien hace lo que quiere. Principalmente incongruentes con la no discriminación y el medio ambiente no se valora ni se respeta. La tolerancia no es adecuada con los valores, nuestro Gobierno no está correctamente establecido.

La Academia Mexicana de Derechos Humanos (1990) nos dice que algunos principios de los DH son la libertad, la justicia y la paz en el mundo, tienen por base el reconocimiento de la dignidad personal y de los derechos iguales e inalienables de todos los miembros de la familia humana. Todos los seres humanos nacen libres e iguales en dignidad y derechos. La realización del ser humano libre necesita condiciones que permitan a cada persona gozar de sus derechos civiles, políticos económicos, sociales y culturales.

Contar con un sistema de protección, promoción y defensa para el desarrollo de los DH ha sido la pugna del ser humano y el poder estatal. La Organización de las Naciones Unidas (ONU, 1945) y muchas más organizaciones intergubernamentales y no gubernamentales, en redes nacionales e internacionales que promueven los DH y velan por su respeto.

En 1990 la Declaración Mundial de la Educación para todos de la UNESCO (Organización de las Naciones Unidas para la Educación, la Ciencia y la Cultura), con el fin de buscar universalización de la educación reconoció la necesidad de suprimir la disparidad educativa particularmente en grupos vulnerables a la discriminación y la exclusión (incluyó niñas, los pobres, niños/ as trabajadores y de la calle, población rural, minorías étnicas, población con discapacidad y otros grupos).

La base de la protección de los DH en México la integran la Constitución General de la República y las Constituciones de los Estados, la primera establece, a juicio de los especialistas, garantías para todos los derechos, ya sean directamente en su texto o en las leyes secundarias derivadas.

Díaz Müller (1992) hace una distinción entre los DH y las garantías constitucionales. Los DH son "principios o ideas universalmente aceptadas y reconocidas" y no se restringen a un estado o país. En cambio, las garantías constitucionales son "el instrumento legal que sirve para la protección de los DH en los ordenamientos de los derechos positivos" (pp. 53-54). Instrumentos 
Revista UniversidAd en DiÁlogo • Vol. 9, N. ${ }^{\circ}$ 1, Enero-Junio, 2019 • 31-46

ISSN 2215-2849 • EISSN: 2215-4752

URL: http://www.revistas.una.ac.cr/index.php/dialogo/index CORREO ELECTRÓNICO: universidadendialogo@una.cr

DOI: https://doi.org/10.15359/udre.9-1.2

de protección en México son: la Procuraduría Federal del Consumidor, la Procuraduría Agraria, la Procuraduría Federal de Protección al Ambiente, la Procuraduría para la Defensa de los Indígenas establecida en Oaxaca en 1987, la Comisión Nacional de DH y la Comisión Estatal de DH en los Estados.

En este sentido, los alumnos de la PA y del Propedéutico proponen algunas ideas para conocer y respetar los derechos humanos y tener bienestar social: los estudiantes de primer año piensan que los valores son cuestiones que debemos practicar todos los días, y se preguntan ¿cómo es que aprendieron los valores en su casa?, ¿cuáles son los valores más importantes?, ¿para qué sirven los DH? Los DH no son como los pintan, algunas veces corresponde bien la gente, otras de lo peor manera, es fundamental que los derechos humanos se inculquen en los niños, solo así se puede transformar a la gente. La comunicación entre familiares es muy importante para aprender valores. ¿Qué hacer en caso de que se violen los DH?, ¿de qué forma responder ante un acto de discriminación?

Los estudiantes de segundo año proponen que se realice alguna reflexión o un cuento, que ponga en contexto lo que significan los DH, y no solo lo que está escrito. Los estudiantes del Propedéutico piensan que no es necesario un reglamento de conducta humana como los $\mathrm{DH}$, deberíamos mejor aprender sobre el amor a la naturaleza y nuestros semejantes.

Los grandes problemas son ocasionados por la pérdida de los valores y el irrespeto a los $\mathrm{DH}$, sobre todo el irrespeto a los indígenas, la preferencia sexual, el color de piel, la etnia o la fisonomía; otros creen que es un tema interesante, lo malo es que es solo teórico.

El primero de los derechos es el derecho a la vida, exige respeto a la integridad de la persona, a su desarrollo pleno, a la sociabilidad necesaria para la satisfacción de las necesidades, respeto a la familia y a otros grupos de socialización, a la naturaleza, etc. La educación tendrá por objeto el pleno desarrollo de la personalidad humana y el fortalecimiento del respeto a los DH y las libertades fundamentales, favoreciendo la comprensión, la tolerancia y la amistad entre todas las naciones y todos los grupos étnicos y religiosos y desarrollando las actividades de las Naciones Unidas para el mantenimiento de la paz (Barba, 2013).

"Toda persona tiene derecho a gozar de un medio ambiente sano y ecológicamente equilibrado para su desarrollo y bienestar; corresponde al Estado garantizar este derecho. Quién ocasione un daño o deterioro ambiental tendrá las responsabilidades que establezcan las leyes". 
URL: http://www.revistas.una.ac.cr/index.php/dialogo/index

CORREO ELECTRÓNICO: universidadendialogo@una.cr

DOI: https://doi.org/10.15359/udre.9-1.2

\section{Conclusión}

Más de la mitad del ingreso a la PA, el 55\%, y más de dos quintas partes del ingreso al Propedéutico, $64.7 \%$, procedieron de entidades de muy alta y alta marginación donde la desigualdad de oportunidades, de participación en el proceso de desarrollo y el disfrute de beneficios se encuentran en situación de desventaja social, desigualdades sociales, particularmente en su bienestar social. La Universidad de Chapingo tiene que proporcionarles una educación de calidad a estos estudiantes que no han logrado acceder a un bienestar social, además de la integración y funcionamiento del sistema educativo y su formación como estudiantes responsables, solidarios, participativos y críticos.

En cualquier intervención didáctica en el aula deben considerarse dos referentes: la función social de la educación en la etapa educativa obligatoria (preparatoria) y las aportaciones de la psicología de la educación sobre el proceso de enseñanza-aprendizaje. En el primer referente es necesario proporcionar al alumno una educación integral, es decir, en conocimientos, destrezas y valores morales en todos los ámbitos de la vida, personal, familiar, social y profesional. Sin embargo, en la PA y el Propedéutico no se les da continuidad a estos valores morales, ni al bienestar social (medio ambiente, tolerancia, violencia), ni siquiera saben cuáles son los $\mathrm{DH}$; tienen la oportunidad de convivir en un grupo heterogéneo, de diferentes culturas y marginados, pueden trabajar juntos, ayudarse, cooperar, conocerse mejor, aceptarse y respetarse mutuamente.

Es necesario realizar un planteamiento sobre la enseñanza actual en DH y bienestar social con miras a nuevas formas de organizar los programas, partiendo de estudios sociológicos del estudiante y del conocimiento y comprensión de los principios que rigen la convivencia en educación moral, a partir de la realidad para la formación de conciencia moral de los alumnos y la formación para la participación, la creación de un ambiente democrático en el salón de clase, la resolución de conflictos, estrategias, compresión de nosotros mismo y de los demás. También se pueden ofrecer materias y foros sobre DH y bienestar social. Algunos programas sobre diversidad étnica, actitudes antidiscriminatorias, la paz, la solidaridad internacional, el humanitarismo, la defensa del medio ambiente, la participación y tolerancia.

En la Universidad de Chapingo los DH y el bienestar social tienen diversas expresiones histórico-culturales, siendo un problema fundamental, por lo que debe promoverse la corrección de estructuras y tendencias políticas, económicas y sociales que no permiten el establecimiento de condiciones 
reales de dignidad e igualdad para las personas. En consecuencia, los profesores no están preparados para trabajar con estudiantes marginados y lidiar con su forma de pensar. Por lo tanto, es necesario dar seguimiento a los estudiantes y profesores sobre el bienestar social y los DH.

El objetivo de este estudio fue mostrar que los estudiantes de la Preparatoria Agrícola y del Propedéutico utilizan los derechos humanos y viven o disfrutan de un bienestar social, no utilizan los $\mathrm{DH}$, tampoco tienen un bienestar, solo lo que aprendieron en casa, no hay realmente un seguimiento, ni estudios sobre este tema.

\section{Referencias bibliográficas}

Academia Mexicana de Derechos Humanos. (1990). Educación Básica y Derechos Humanos. México.

Barba, J. B. (2013). Educación para los derechos humanos. México: Fondo de Cultura Económica.

Consejo Nacional de Población, CONAPO. (2010). Boletin no. 21/2010. Indicadores sobre condiciones de vida de jóvenes índigenas. En: www. conapo.gob.mx/prensa/2010/bolo20.2010.pdf

Díaz, L. (1992). Manual de derechos humanos (2da. ed.). Comisión Nacional de Derechos Humanos, México

Hernández, J. L. (2013). Informe del Departamento de Preparatoria Agrícola de la UACh. Universidad Autónoma Chapingo, México.

Hernández, M. T. y Fuentes, D. (1991). Hacia una cultura de los derechos humanos. Comisión Nacional de Derechos Humanos, México.

Mata, A. y Villar, L. (1993). Ira. Parte, diagnóstico, formación docente, propuestas curriculares. Universidad Autónoma Chapingo, México.

Padilla, N. E. (2013). La educación en derechos humanos en México en los niveles preescolar, primaria y secundaria. Universidad Iberoamericana, México.

Pérez, S. M., Livera, M. R. y Carranza, A. E. (2010). Perfil de ingreso 2010. Universidad Autónoma Chapingo, México.

Unidad de Planeación, Organización y Método (UPOM) (2010). Anuario estadístico. Universidad Autónoma Chapingo. 